\title{
Sensitivity of bimodal listeners to interaural time differences with modulated single- and multiple-channel stimuli
}

Running title: Bimodal ITD perception

Key words: bimodal stimulation, cochlear implant, hearing aid, interaural time difference

Tom Francart ${ }^{1}$

Exp ORL, Dept. Neurosciences, K.U.Leuven

Anneke Lenssen ${ }^{2}$

Exp ORL, Dept. Neurosciences, K.U.Leuven

Jan Wouters ${ }^{3}$

Exp ORL, Dept Neurosciences, K.U.Leuven

Corresponding author:

Tom Francart

Exp ORL, Dept. Neurosciences, K.U.Leuven,

$\mathrm{O} \& \mathrm{~N} 2$,

Herestraat 49 bus 721 ,

B-3000 Leuven, Belgium

tel: $+32(0) 16330476$

fax: +32 (0)16330486

tom.francart@med.kuleuven.be

\footnotetext{
${ }^{1}$ tom.francart@med.kuleuven.be

2 anneke.lenssen@med.kuleuven.be

3 jan.wouters@med.kuleuven.be
} 


\section{List of acronyms}

CI cochlear implant

HA hearing aid

ILD interaural level difference

ITD interaural time difference

JND just noticeable difference

NH normal hearing 


\begin{abstract}
In a previous study it was shown that users of a cochlear implant and a contralateral hearing aid are sensitive to interaural time differences (ITDs). In the current study we investigated (1) the influence on ITD sensitivity of bilaterally varying the place of excitation in the cochlea and of modulation frequency, and (2) sensitivity to ITD with a 3-channel stimulus generated using CIS-like processing. The stimuli were (1) a high-frequency carrier (acoustic sinusoid and single-electrode electric pulse train), modulated with a half wave rectified low frequency sinusoid (a so-called transposed stimulus), and (2) a 3-channel stimulus, generated by sending an acoustic click train through processing similar to the CIS strategy. Four bimodal listeners were sensitive to ITD for both stimulus types. For the first stimulus type, there was no significant influence on ITD sensitivity of acoustic carrier frequency. Performance decreased with increasing modulation frequency with a limit of sensitivity at around 150-200 Hz. Sensitivity was similar for the single- and 3-channel stimulus. The results indicate the possibility of ITD perception with adapted clinical processors, which can lead to improved sound source localization and binaural unmasking.
\end{abstract}


1

\section{Introduction}

For cochlear implant (CI) users with residual hearing in the non-implanted ear, combined electric and acoustic stimulation is a common alternative to bilateral cochlear implantation. This configuration is called bimodal stimulation. As CI users sometimes perform better than severely impaired hearing aid (HA) users, implantation criteria are changing, leading to an increasing population of CI users with significant residual hearing in the non-implanted ear. With clinical devices it has been demonstrated that a contralateral hearing aid improves sound source localization and speech perception performance in unilaterally implanted subjects, as illustrated in a recent review by Ching et al. [2007]. While localization performance improves by adding a contralateral HA, it is still poor compared to normal hearing (NH) listeners [Ching et al., 2001, 2004, 2005, Seeber et al., 2004, Tyler et al., 2007].

In previous studies, we demonstrated that bimodal listeners are sensitive to interaural level differences (ILDs) and interaural time differences (ITDs), which are the main cues for localization in the horizontal plane [Francart et al., 2008a, 2009]. In the current study, we further investigated ITD sensitivity of bimodal listeners with more ecologically relevant stimuli, to bridge the gap between pure laboratory experiments and application in clinical speech processors.

The ITD is an important cue for sound localization. Sensitivity to ITD is related to binaural unmasking of a target sound in a spatially separated masker [Colburn et al., 2006]. For a human head, ITDs range from $0 \mu \mathrm{s}$ for a sound straight ahead to $690 \mu \mathrm{s}$ for a sound at $90^{\circ}$ [Feddersen et al., 1957]. For high frequency sounds, the ITD becomes ambiguous, because the period is smaller than the available ITD. In that case, however, the auditory system can make use of ITDs in the envelope [Henning, 1974].

Changes in ITD of about $10 \mu$ s can be detected by NH subjects in low frequency sinusoids [Yost, 1974]. Above about $1500 \mathrm{~Hz}$ this process breaks down [Yost et al., 1971]. At high frequencies, ITDs can be detected in the onset (transient part) or in the ongoing envelope part of a time-varying signal [Henning, 1974]. Buell et al. [2008] have shown that sensitivity to ITD in the ongoing part is better than to ITD in the onset. If an ITD is present in both the temporal fine structure and the envelope, NH listeners are sensitive to both, but the fine structure ITD is dominant [Bernstein and Trahiotis, 1985].

Van de Par and Kohlrausch [1997] introduced the concept of the "transposed" signal. A transposed signal is generated by multiplying a high-frequency carrier by a half-wave rectified low-frequency signal (e.g., a low-frequency pure tone). If desired, the frequency content of the resulting signal can be limited by low-pass filtering the low-frequency signal after half-wave rectification. While ITD performance with amplitude modulated high frequency sinusoids is generally worse than with pure tones, performance with transposed stimuli is nearly at the same level as with pure tones [Bernstein and Trahiotis, 2002]. They attributed this to the peripheral processing of the auditory system: after envelope detection by half-wave rectification and low pass filtering, a transposed stimulus yields the same envelope shape as a low frequency sinusoid, while a sinusoidally amplitude modulated stimulus yields a different envelope shape. Recent studies show that the main factors involved are the temporal properties of the envelope, such as steepness of the onsets and the duty cycle [Bernstein and Trahiotis, 2009, Ewert et al., 2009, Laback et al., 2009].

When the carriers of modulated signals are interaurally discrepant, just noticeable differences (JNDs) in ITD can still be measured, but performance decreases rapidly with increasing interaural frequency difference [Nuetzel and Hafter, 1981]. However, Blanks et al. [2007] have shown that for a simpler psychophysical task or an animal model, there is sensitivity to ITD when the interaural frequency difference increases up to several octaves. In bilateral CI 
listeners, there is ITD sensitivity for much larger interaural differences in place of stimulation in the cochlea [Poon et al., 2009].

Different studies have shown that bilateral CI listeners are sensitive to ITDs in single-electrode pulse trains for pulse rates up to around 800 pps (e.g., Majdak et al. [2006], van Hoesel [2007], van Hoesel and Tyler [2003], van Hoesel et al. [2009]), with large inter-subject variability. In these studies, stimuli were presented under direct computer control, without using clinical speech processors.

It has also been shown that bilateral CI listeners are sensitive to ITDs in the envelope imposed on a high-rate pulse train, with a rate limit similar to or marginally lower than with unmodulated low-rate pulse trains [Majdak et al., 2006, van Hoesel, 2007, van Hoesel and Tyler, 2003, van Hoesel et al., 2009]. However, JNDs, are higher for modulated signals [van Hoesel, 2007, van Hoesel et al., 2009]. Several studies have also found that performance increases with increasing peak amplitude [Laback et al., 2009, van Hoesel, 2007]. In very recent work, it has been shown that the envelope shape is an important determinant for ITD detection performance. Using trapezoidal envelopes, Laback et al. [2009] found that the duty cycle had the largest influence. They also demonstrated that the envelope slope had no influence on performance in $\mathrm{CI}$ listeners, while it did have a significant effect in NH listeners.

We are not aware of any studies that investigate bilateral CI ITD sensitivity with multi-channel stimuli under direct computer control. However, several studies have investigated ITD sensitivity with clinical processors, stimulating multiple electrodes [Grantham et al., 2008, Laback et al., 2004, Senn et al., 2005]. Performance is generally lower with speech processors than with direct stimulation. It remains the question whether this is due to the simultaneous ${ }^{4}$ stimulation of more than one electrode, or due to the signal processing in the speech processors.

The influence of the speech processor is evident from the fact that ITD performance is very stimulus dependent. Performance with clinical processors is best for (acoustic) click trains, worse for noise bursts [Senn et al., 2005], and even worse for a speech fragment or speech modulated noise [Laback et al., 2004]. With clinical processors, bilateral CI listeners mainly use ILD cues for localization [Seeber and Fastl, 2008, van Hoesel, 2004]. While binaural advantages are apparent, they are mainly due to the better-ear-effect. This is in contrast to the direct-stimulation studies where perception of both binaural cues is shown.

In our previous ITD perception study with bimodal listeners [Francart et al., 2009], the stimulus was a low-rate (100 pps) pulse train. In the current study we investigated sensitivity to ITD with stimuli more similar to those produced by clinically used speech processors. We performed 3 different experiments. In experiment 1 , we assessed the influence of bilaterally matching the place of excitation in the cochlea. In experiment 2 we assessed the influence of modulation frequency on ITD sensitivity to evaluate the plausibility of using ITD cues in everyday sounds, such as spoken vowels. In experiment 1 and 2 we used a transposed stimulus with a high-rate carrier (900 or $6300 \mathrm{pps)}$ on a single channel. The transposed stimulus was selected because NH subjects have good ITD sensitivity with transposed stimuli and because it is similar to the electrical pulse output of a clinical processor on a single channel, as response to an acoustically presented vowel. Finally, in experiment 3, we assessed ITD sensitivity with 3 electrodes stimulated simultaneously. The 3-channel stimulus was designed to assess the effect of channel interactions and the generalizability of the single-channel psychophysical data to multi-channel stimulation in clinical speech processors. Due to spread of excitation, channel interactions might occur that could degrade ITD sensitivity.

\footnotetext{
${ }^{4}$ Note that in most clinically used processors electrodes are not stimulated simultaneously in the strictest sense, but sequentially with very small inter-pulse intervals.
} 


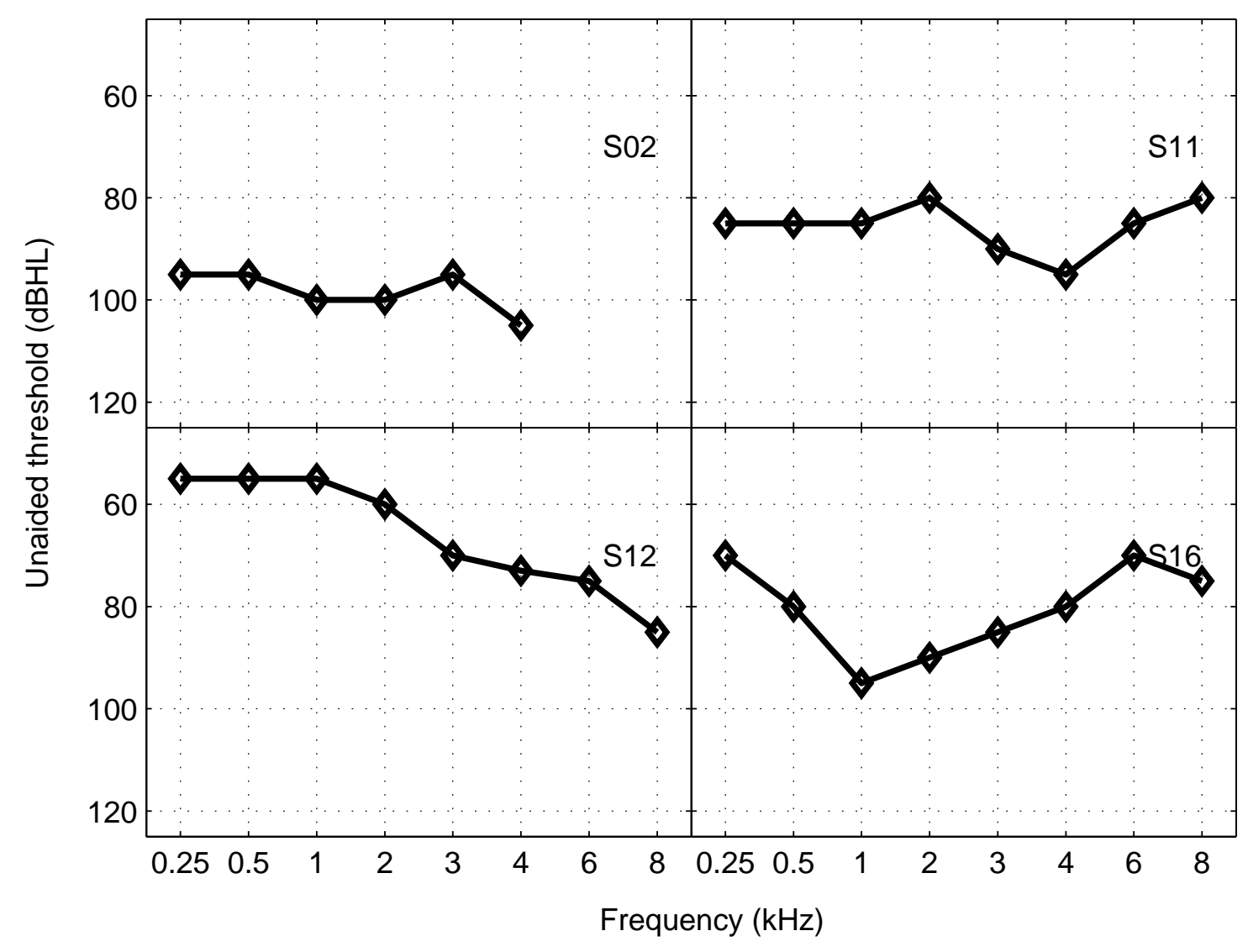

Figure 1: Pure tone unaided audiograms as measured during routine audiometry. Note that the vertical axis starts at $50 \mathrm{dBHL}$.

\section{Methods}

\section{$2.1 \quad$ Subjects}

Four subjects were recruited from the clinical population of the University Hospital Maastricht (AZM) and the University Hospital Leuven (UZLeuven). They were volunteers and signed an informed consent form. This study was approved by the local medical ethical committees. They wore a HA contralaterally to their CI on a daily basis. S12 and S16 had an electrode array of the Contour Advance type; the other subjects had an array of the Contour type. The clinical processors were of the Freedom type. Their unaided pure tone audiograms as measured during routine audiometry are shown in figure 1. Various subject information is listed in table I.

Subjects S02, S11 and S12 also participated in our previous bimodal ITD study [Francart et al., 2009].

\subsection{Apparatus}

The subjects' own HAs or speech processors were not used. All stimuli were presented under direct computer control using the APEX 3 program developed at ExpORL (K.U.Leuven)

[Francart et al., 2008b]. For acoustic stimulation, we used an RME Multiface II sound card, 


\begin{tabular}{lllllll}
\hline Subject & Age & M of use & CI side & Etiology & Electrode exp 1\&2 & Electrodes exp 3 \\
\hline S02 & 66 & 65 & R & Noise exposure & 11 & $9,10,11$ \\
S11 & 62 & 41 & R & Meniere & 16 & $14,15,16$ \\
S12 & 65 & 21 & L & Genetic (DFNA9) & 11 & $9,10,11$ \\
S16 & 70 & 8 & L & Progressive & 16 & $14,15,16$
\end{tabular}

Table I: Subject information: "Age" is in years at the time of testing. "M of use" is the number of months of implant use at the time of testing. "CI side" is left $(\mathrm{L})$ or right $(\mathrm{R})$, the HA was on the other side. The two rightmost columns show the tested electrodes in respectively experiment 1 and 2 , and experiment 3 .

1 connected to a single insert phone of type Etymotic ERA 3A. For electric stimulation, we used the Cochlear NICv2 interface, connected to an L34 experimental processor provided by Cochlear Ltd. To ensure synchronous stimulation, the L34 was set up to start stimulating when a trigger pulse was received from the sound card. In this way synchronous stimulation was achieved with $200 \mathrm{~ns}$ accuracy. The relative position of the acoustic and electric signal was calibrated using the output of an implant-in-a-box and the electric output of the sound card visualized on an oscilloscope. It was calibrated such that that the first edge of the electric pulse coincided with the top of the first peak of an acoustic click. From the reported interaural delay values, the delay of the insert-phone $(1154 \mu \mathrm{s})$ was removed. The insert phone was calibrated using a 2cc coupler conforming to the ISO389 norm. The shapes of both the electric and acoustic signals were checked using an oscilloscope.

\subsection{Stimuli}

All electric pulses were presented in monopolar mode $(\mathrm{MP} 1+2)$, with a biphasic pulse shape. The phase width was $25 \mu \mathrm{s}$ and the inter phase gap was $8 \mu \mathrm{s}$. All stimuli were in total $1 \mathrm{~s}$ long and were generated using custom Matlab scripts. The used electrodes are shown in table I. Electrodes are numbered from apex to base, i.e., electrode 1 is the most apical electrode (normally corresponding to the lowest place pitch) and electrode 22 is the most basal electrode. To achieve maximal sensitivity to the stimulus parameters under investigation, we selected an electrode that was expected to yield good ITD sensitivity for each subject. For experiment 1 and 2 , initially electrode 11 was selected, but if performance was poor, electrode 16 was selected. For S02, S11 and S12 this decision was based on the results from our previous study [Francart et al., 2009] and for S16 some preliminary experiments were done. For experiment 3, either electrodes 9, 10 and 11 were selected, or electrodes 14,15 and 16, corresponding to the electrode selected in experiment 1 and 2 .

Two different stimulus types were used, a single channel transposed stimulus (experiment 1 and 2) and a filtered click train processed by a 3-channel CIS-like system (experiment 3). A stimulus is the combination of an electric signal and an acoustic signal, presented simultaneously to the two ears of the subject.

An example transposed stimulus is shown in figure 2. The acoustic signal was generated by modulating a sinusoidal carrier (with frequency $f_{c}$ ) with a half-wave rectified low frequency sinusoidal modulator (with frequency $f_{m}$ ). The electric signal was generated by modulating a high-rate (900 or $6300 \mathrm{pps}$ ) pulse train with the same modulator. An overview of the different stimulus parameters per experiment is shown in table II.

In experiment 1 the influence of carrier frequency on single-channel ITD sensitivity was assessed, 

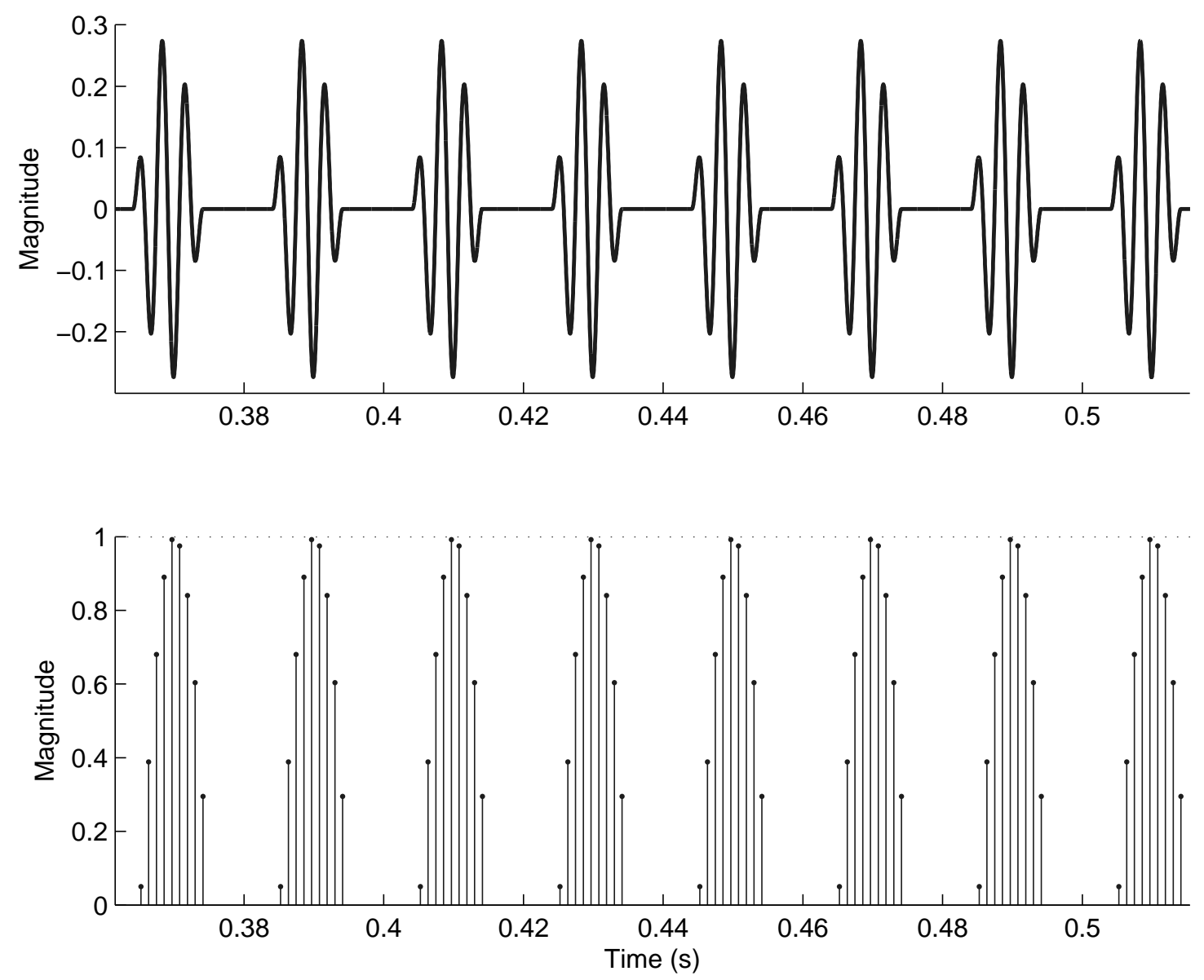

Figure 2: Fragment of an example transposed stimulus. The top panel shows the acoustic signal and the bottom panel shows the pulses on a single electrode. The modulation frequency $\mathrm{f}_{\mathrm{m}}=50 \mathrm{~Hz}$, the acoustic carrier frequency $\mathrm{f}_{\mathrm{c}}=300 \mathrm{~Hz}$ and the electric carrier was a pulse train of $900 \mathrm{pps}$

\begin{tabular}{lcccc}
\hline Experiment & $f_{m}(\mathrm{~Hz})$ & El carrier $(\mathrm{pps})$ & $f_{c}(\mathrm{~Hz})$ & Ramping \\
\hline 1 & 50 & 900 & $300-3600$ & \\
2 & $50-300$ & 6300 & 1200 & $\checkmark$ \\
3 & 50 & 900 & $1248,1432,1682$ & $\checkmark$ \\
\hline
\end{tabular}

Table II: Stimulus parameter overview. $f_{m}$ is the modulation rate, "El carrier" is the carrier rate of the electric signal, $f_{c}$ is the carrier frequency of the acoustic signal. For experiment 3 , the given acoustic carrier frequencies, are the center frequencies of the analysis filter bank. A $\checkmark$ in "ramping" indicates that ramping of $50 \mathrm{~ms}$ was applied. 


\section{Click train of $50 \mathrm{~Hz}$ filtered $1000-1750 \mathrm{~Hz}$}

\section{Bandpass Envelope Pulse filtering detection generation}

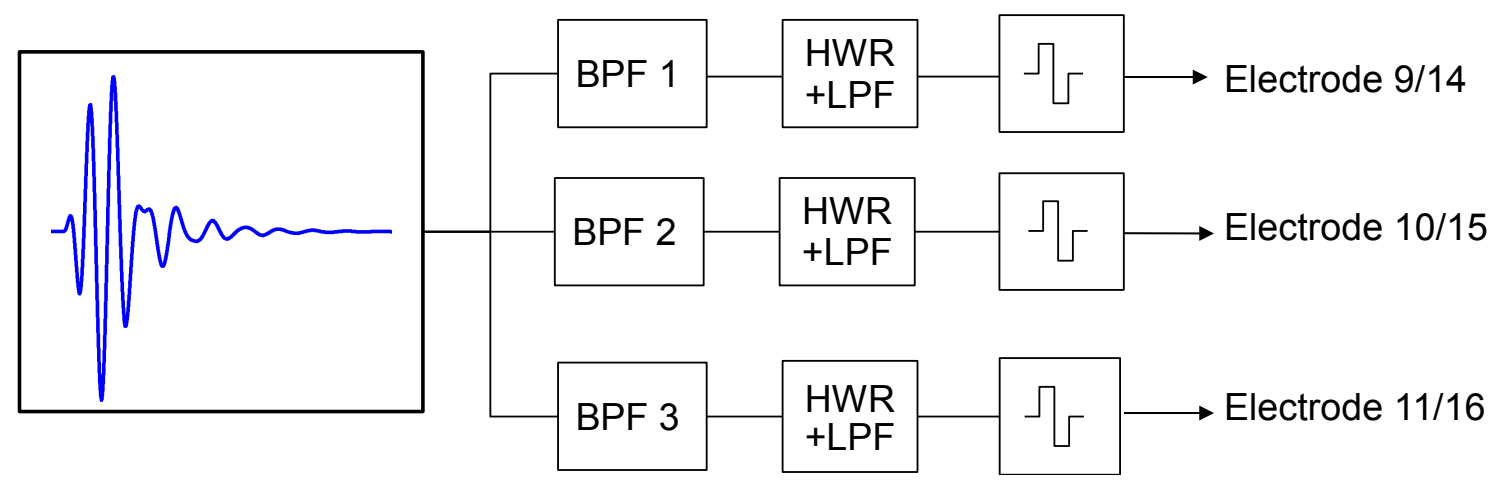

Figure 3: CIS-like signal processing, as used in experiment 3

keeping all other parameters constant. In this way, the influence of bilaterally varying the place of excitation on ITD sensitivity is assessed. In experiment 2, the influence of modulation frequency on ITD sensitivity is assessed. In experiment 3 , the influence of simultaneous stimulation of multiple electrodes on ITD perception is assessed.

In experiment 1 , the modulation frequency $\left(f_{m}\right)$ was fixed to $50 \mathrm{~Hz}$ and the acoustic carrier frequency $\left(f_{c}\right)$ was either $300,600,1200,2400$, or $3600 \mathrm{~Hz}$. The electric carrier was a pulse train of 900 pps. In experiment 2, the acoustic carrier frequency was fixed to $1200 \mathrm{~Hz}$ and the modulation frequency was one of 50,100, 200 and $300 \mathrm{~Hz}$. The electric carrier was a pulse train of 6300 pps (in experiment 2 a higher electric carrier rate was selected to avoid aliasing at higher modulation frequencies). In experiment 1 , no ramping was applied, in experiment 2 , cosinusoidal ramping of $50 \mathrm{~ms}$ was applied to the onset and offset of the electric and acoustic signal.

The acoustic part of the 3-channel stimulus was a band limited click train with a repetition rate of $\mathrm{F}_{0}=50 \mathrm{~Hz}$, filtered between 1000 and $1750 \mathrm{~Hz}$ with a 4th order Butterworth filter, with $50 \mathrm{~ms}$ cosinusoidal ramping applied to the onset and offset. The electric signal was generated by sending the acoustic signal through CIS-like signal processing, as shown schematically in figure 3 . The "acoustic" signal was first sent through a 4th order Butterworth filter bank (BPF 1,2,3) with cut-off frequencies similar to the cut-off frequencies used in the clinical ACE filter bank for electrode 9, 10 and 11 . Then envelope detection was done by half-wave rectifying (HWR) and low pass filtering (LPF) (4th order Butterworth filter with a cut-off frequency of $300 \mathrm{~Hz}$ ) the signals in each channel. Then a pulse train of 900 pps was modulated with the signal in each channel and sent to the electrodes shown in table II. The pulse timing on the different electrodes was chosen as to maximize the time in between pulses, i.e., there was always a time of $1 /(3 * 900) \mathrm{s}=370 \mu \mathrm{s}$ between each two sequential pulses (on different electrodes). In figure 4 the spectrum of the filtered click train and the frequency response of the three band pass filters are shown. In figure 5 an excerpt of an example 3-channel stimulus is shown.

\subsection{Procedure}

Each stimulus was first set to a comfortable level and balanced in level. Then the JND in ITD was determined with a single interval two alternatives forced choice constant stimuli procedure.

The level balancing procedure was based on extent of lateralization; if the stimulus could be 


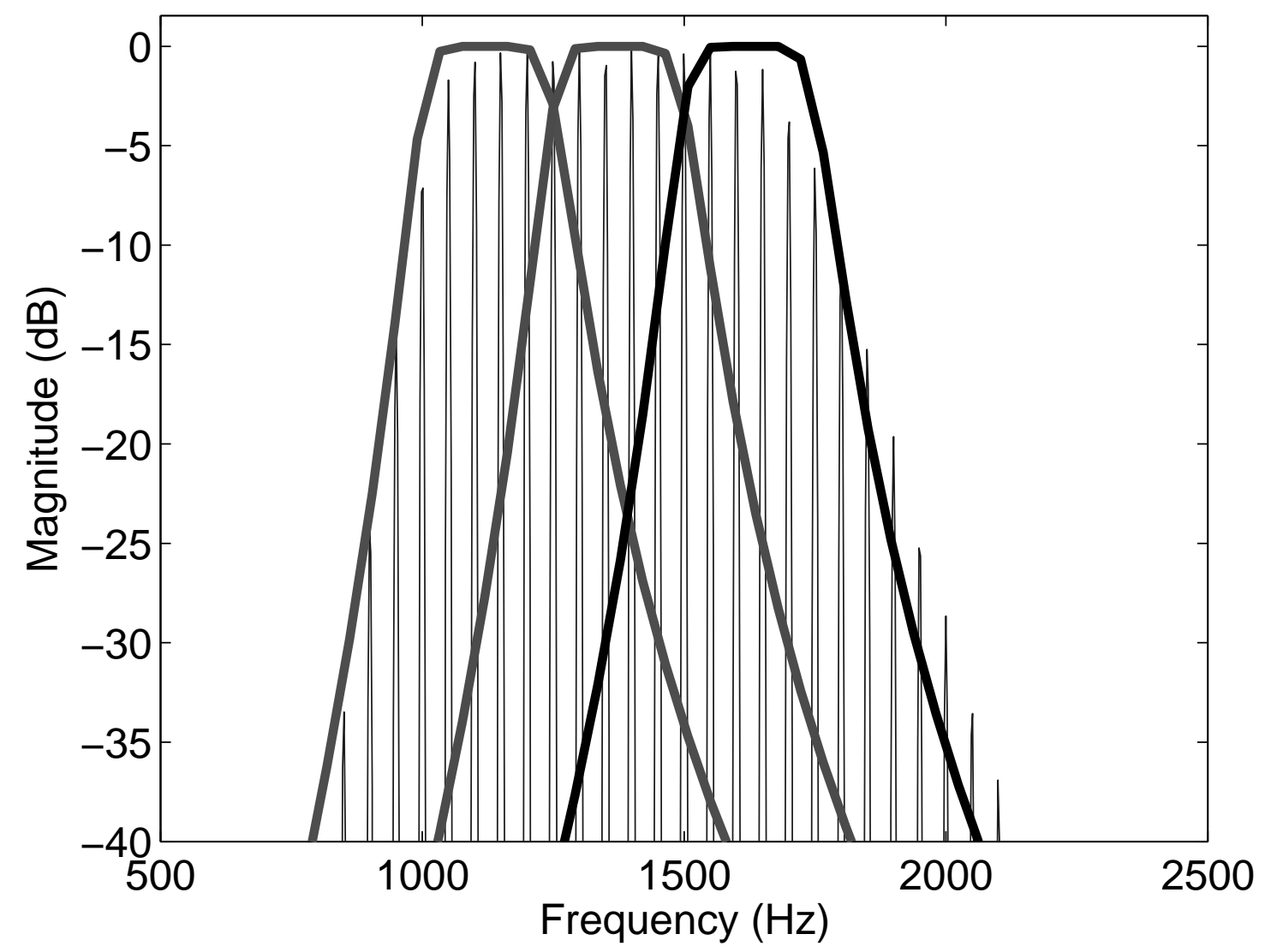

Figure 4: Spectrum of the filtered click train and the frequency response of the three used band pass filters. 

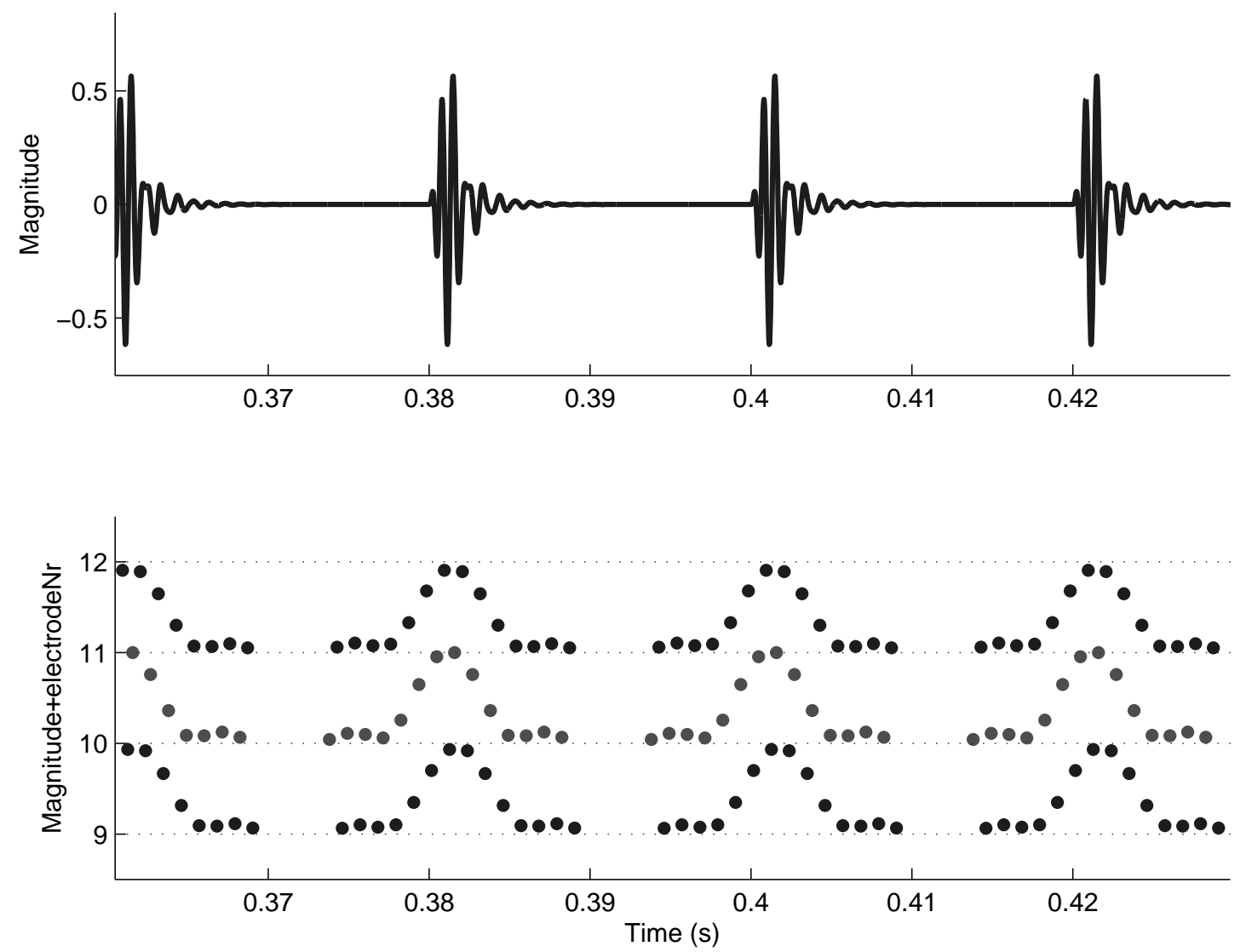

Figure 5: Fragment of an example 3-channel stimulus, as used in experiment 3. The top panel shows the acoustic signal. The bottom panel shows the electric signal. Only the magnitude of the pulses is shown. 
$f_{m}=50 \mathrm{~Hz}$

\begin{tabular}{rccccc}
\hline Subject $/ f_{c}$ & $300 \mathrm{~Hz}$ & $600 \mathrm{~Hz}$ & $1200 \mathrm{~Hz}$ & $2400 \mathrm{~Hz}$ & $3600 \mathrm{~Hz}$ \\
\hline $\mathrm{S} 02$ & & 113 & 117 & 115 & 116 \\
$\mathrm{~S} 11$ & & 108 & 101 & 105 & 114 \\
$\mathrm{~S} 12$ & 93 & 82 & 87 & 96 & 116 \\
$\mathrm{~S} 16$ & & 104 & 105 & 102 & \\
\hline
\end{tabular}

$f_{c}=1200 \mathrm{~Hz}$

\begin{tabular}{rcccccc}
\hline Subject $/ f_{m}$ & $50 \mathrm{~Hz}$ & $100 \mathrm{~Hz}$ & $150 \mathrm{~Hz}$ & $200 \mathrm{~Hz}$ & $300 \mathrm{~Hz}$ & multichannel \\
\hline $\mathrm{S} 02$ & 117 & 114 & 116 & 116 & & 110 \\
$\mathrm{~S} 11$ & 101 & 100 & 100 & & & 99 \\
$\mathrm{~S} 12$ & 87 & 91 & 91 & 94 & 94 & 86 \\
$\mathrm{~S} 16$ & 105 & 101 & 101 & & & 101 \\
\hline
\end{tabular}

Table III: Average broadband acoustic level per subject and stimulus type, all in dB SPL.

steered equally far to either side of the head by varying only ITD, it was considered balanced in level $^{5}$. First the acoustic signal was set to a level that was perceived as "good" on a 7-point scale ranging from "inaudible" to "uncomfortably loud". Then the electric signal was set separately to a level perceived as "good". Then the stimulus was presented binaurally with an ITD that was expected to approximately correspond to what would be ITD 0 in normal hearing subjects, i.e., the electric signal was delayed by $1.5 \mathrm{~ms}$ relative to the acoustic signal [Francart et al., 2009]. Then the intensity of the acoustic or electric signal was slightly adjusted to achieve a centred sound image. Thereafter, it was attempted to shift the sound image to either side of the head by changing the ITD. If the maximal extent of laterality was perceived similar for each side of the head, the level was considered balanced. Otherwise, the balance was adjusted and the latter test was repeated. The average acoustic levels used for each subject and stimulus are shown in table III.

To determine the JND in ITD, the psychometric function for ITD was estimated using a single interval, two alternatives forced choice constant stimuli procedure. In a single run, a number of ITDs was selected over a certain range and a stimulus containing each ITD was presented three times. The subject had to indicate whether the sound was lateralized to the left or right side. The ITDs to be presented in a single run were determined by the experimenter based on previous subject performance. Some very large ITDs (up to $1.5 \mathrm{~ms}$ off-center) were always included to motivate the subject. In the proximity of the crossover point, the intervals were either 500, 250 or $100 \mu \mathrm{s}$, based on the subject's performance. In figure 6 an example psychometric function is shown.

Psychometric functions were fitted to the results using the psignifit toolbox version 2.5.6 for Matlab (see http://bootstrap-software.org/psignifit/) which implements the maximum-likelihood method described by Wichmann and Hill [2001]. The $68 \%$ confidence intervals around the fitted values were obtained by the $\mathrm{BC}_{\mathrm{A}}$ bootstrap method implemented by psignifit, based on 1999 simulations. Results of a psychometric function were only regarded as valid if a confidence interval could be calculated by the bootstrap method and if there was no perfect separation, i.e., if there were points on the slope of the psychometric function different

\footnotetext{
${ }^{5}$ While in our previous study [Francart et al., 2009] a more extended loudness balancing procedure was used, the same extent-of-lateralization-procedure was used there as verification and the results always corresponded very well to the extended procedure.
} 


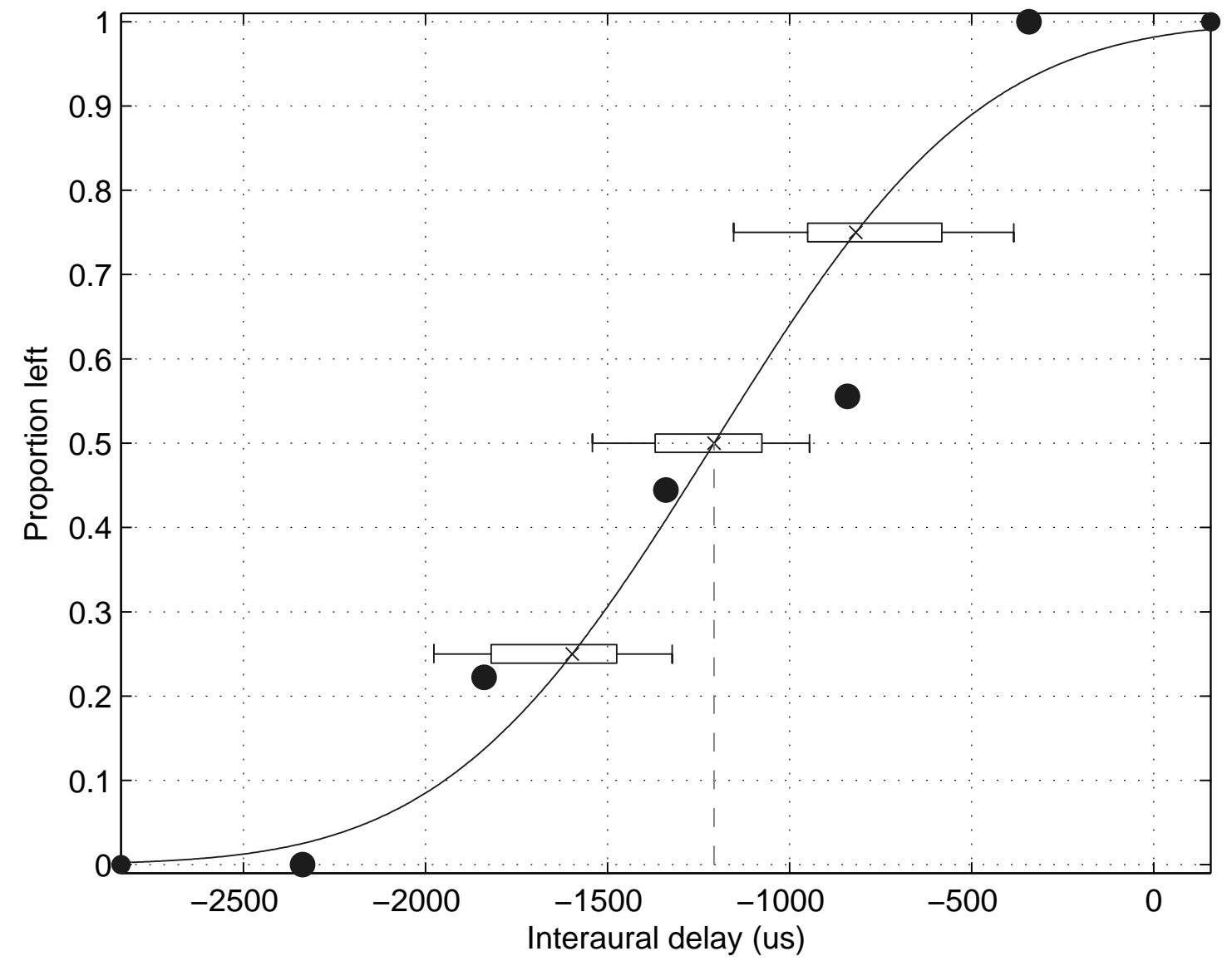

Figure 6: Example psychometric function. In this case the interaural delay for a centered percept was $1208 \mu$ s and the JND was $362 \mu$ s. 
from 0 and 1. Multiple runs of the same condition (stimulus type) were performed during one test session, and the results of those runs were merged into a single psychometric function.

From each psychometric function, the JND in ITD was determined from the slope at $50 \%$ correct as the change in ITD necessary for a $25 \%$ change in performance. The interaural delay that leads to a centred percept $(D)$, i.e., the interaural delay necessary to compensate for the acoustic travelling wave delay, was determined from the interaural delay at $50 \%$ correct. If multiple psychometric functions were determined for the same subject and condition (e.g., during different test sessions), the global JND was calculated by shifting each of the functions such that their estimated $50 \%$ point was $0 \mu$ s and then performing a new estimation of the function (and its corresponding JND) formed by the combination of all (shifted) data points ${ }^{6}$. As a result, the reported JNDs in ITD are each based on a total number of trials ranging from 42 to 297 , with a median of 107 . Conditions were considered significantly different if there was no overlap between the error bars determined by the bootstrap method.

\section{Results and discussion}

For all stimuli that yielded sensitivity to ITD, the subjects informally reported a compact and fused percept. S12 and S02 reported a compact image moving at the back of the head with changing ITD. For conditions that yielded good ITD sensitivity, the image could be shifted entirely to the left or right hand side, changing only ITD.

Besides the JND in ITD, the delay of the electric signal needed to compensate for the acoustic travelling wave delay $(D)$ could be determined. While one would expect this delay to be frequency-dependent, this is not obvious from our data, due to the subjects' limited ITD sensitivity and the amount of data collected. In figure 7 the pooled data are shown for the 4 subjects, for the single-channel experiments (1 and 2) and the 3 -channel experiment (3). We did observe inter-subject differences in the order of $200 \mu \mathrm{s}$. A paired t-test indicates a significant difference of stimulus $(p<0.04)$. This may be due to a change in interaural difference in slope of the envelope [Laback et al., 2009].

\section{$3.1 \quad$ Experiment 1}

In experiment 1 the influence of acoustic carrier frequency $\left(f_{c}\right)$ on ITD sensitivity was assessed while holding all other stimulus parameters constant, thereby evaluating the importance of a bilaterally matched place of excitation in the cochlea. The results are shown in figure 8. Missing bars indicate that there was no sensitivity to ITD for that carrier frequency. There were no significant intra-subject differences in performance between any of the acoustic carrier frequencies for which ITD sensitivity could be measured, except for S12 at $2400 \mathrm{~Hz}$.

The temporal fine structure of the electric and acoustic signals was different: the electric carrier was a high-rate pulse train of $900 \mathrm{pps}$, which is beyond all limits of ITD sensitivity in electric hearing. The acoustic carrier was a sinusoid of a different frequency $(300-3600 \mathrm{~Hz})$, which, except for $300 \mathrm{~Hz}$, was no divisor of the electric pulse rate. It seems therefore very unlikely that fine structure ITD cues were used in the current study ${ }^{7}$. Moreover, in our previous study

\footnotetext{
${ }^{6}$ Note that data from different test sessions were not simply merged into a psychometric function because fluctuations in residual hearing may lead to differences in level balance and potentially small differences in $D$. Merging data with different $D$-values would lead to an apparent increase in JND. Generally, the difference in estimated $50 \%$ point for different measurements of the same condition was in the order of 1 JND or smaller.

${ }^{7}$ Note that in electrical stimulation there is no distinction between temporal fine structure and envelope at the
} 


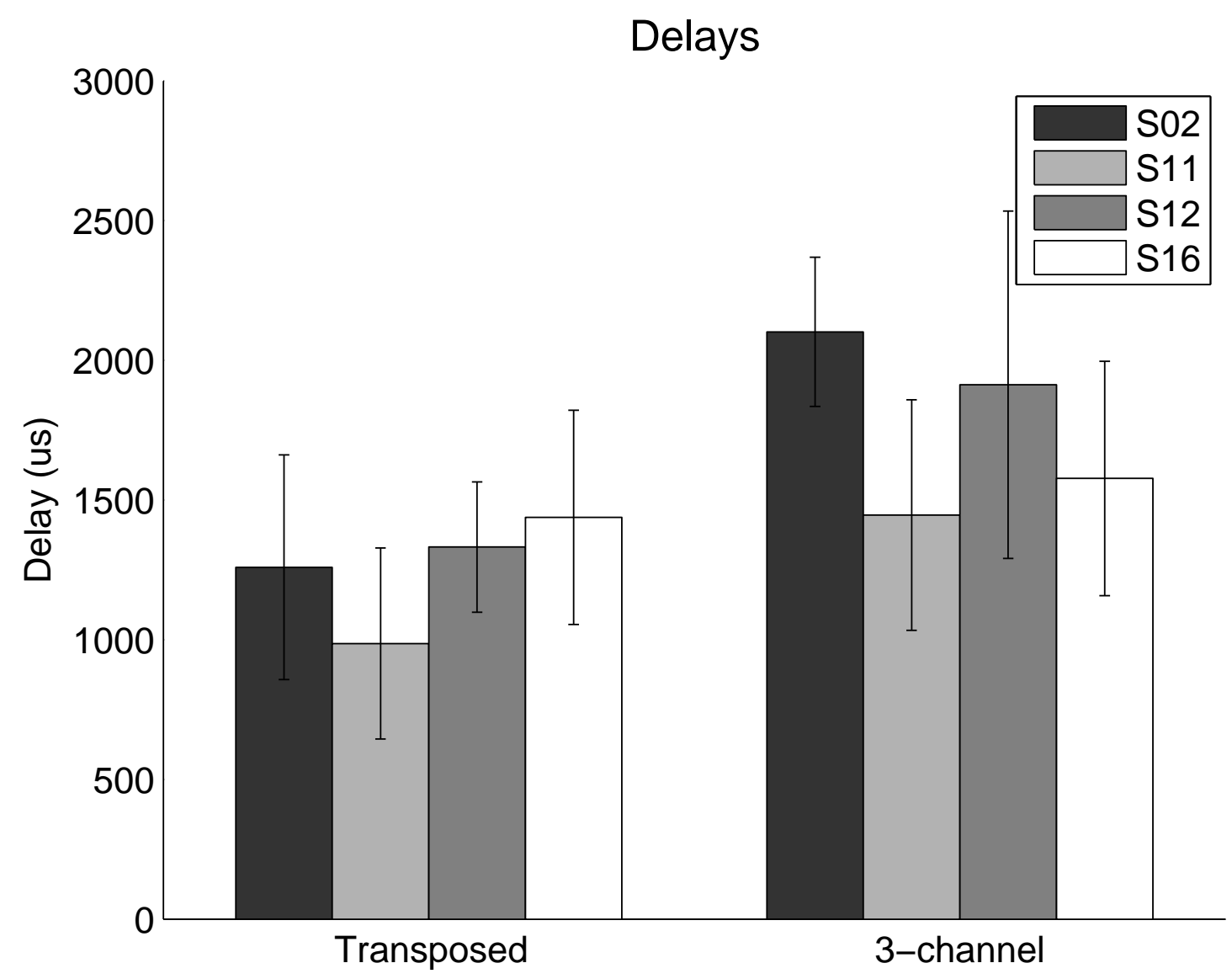

Figure 7: Median delay of the electric signal needed to compensate for the acoustic travelling wave delay $(D)$. The results were pooled per subject over experiment $1 / 2$ and 3 . The error bars indicate inter-quartile ranges. 


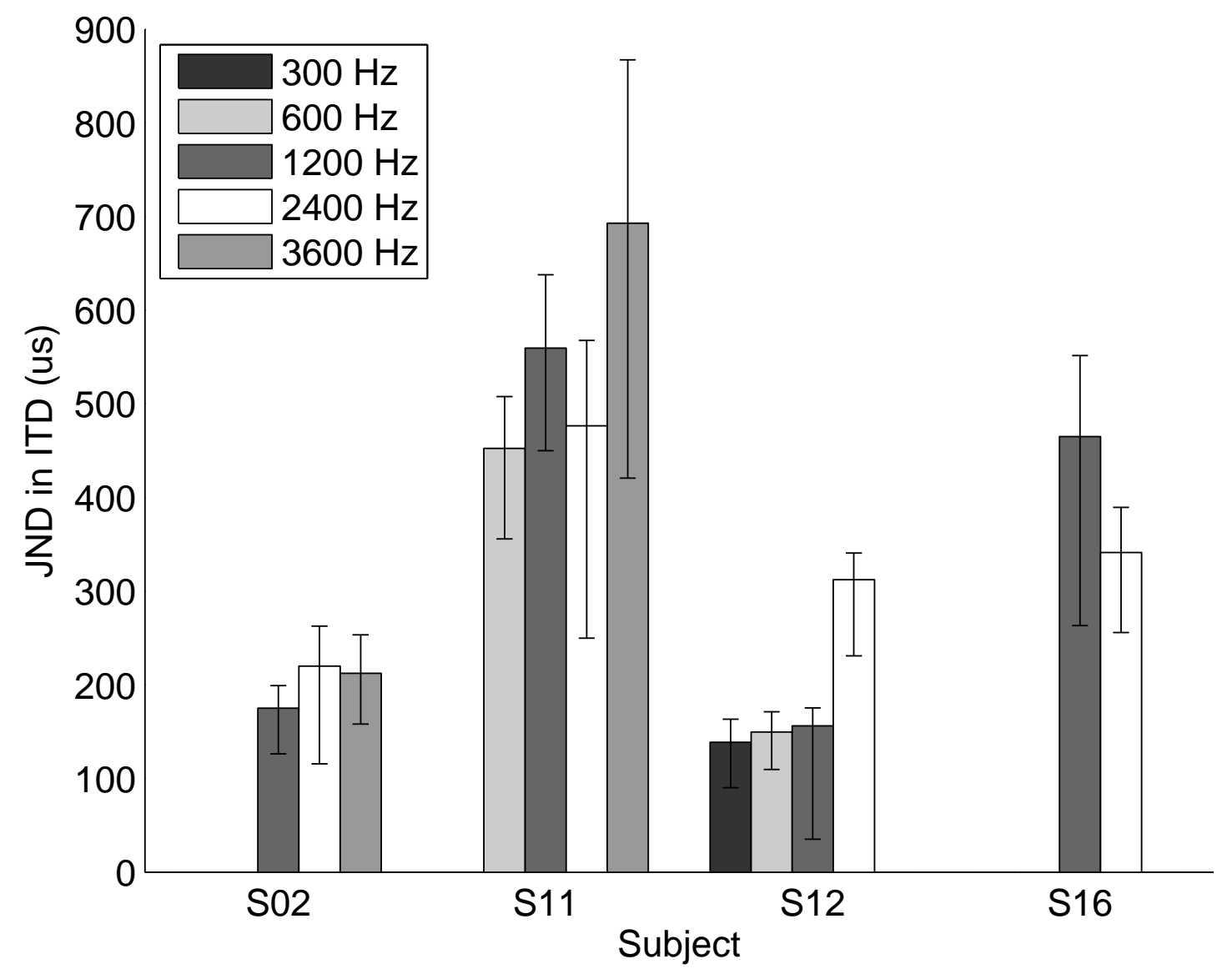

Figure 8: Results of experiment 1 . The modulation frequency $\mathrm{f}_{\mathrm{m}}=50 \mathrm{~Hz}$ and the electric carrier was a pulse train of 900 pps. The carrier frequency $\left(f_{c}\right)$ is shown in the inset. 


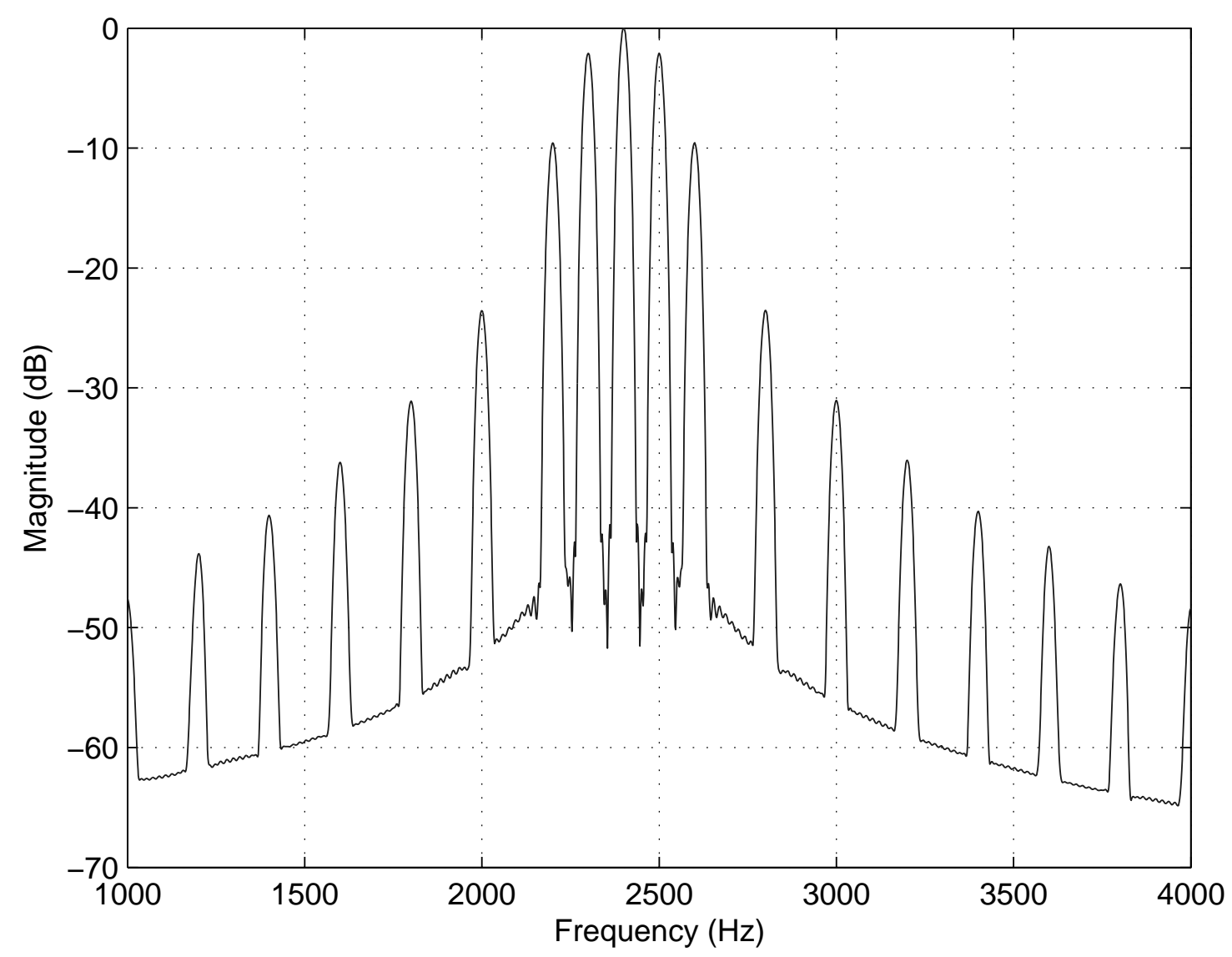

Figure 9: Spectrum of an example transposed stimulus with $\mathrm{f}_{\mathrm{c}}=2400 \mathrm{~Hz}$ and $\mathrm{f}_{\mathrm{m}}=100 \mathrm{~Hz}$.

1 [Francart et al., 2009] we found that with the used stimuli the subjects were not sensitive to 2 ITDs in the temporal fine structure. Therefore the subjects must have used onset or ongoing 3 envelope cues.

4 In our previous study [Francart et al., 2009], for each electrode we found a non-significant tendency of best performance for a certain acoustic frequency. In the current experiment 1 we investigated this further. There were no significant differences between different conditions $\left(f_{c}\right)$ where the subjects were sensitive to ITD. This low sensitivity to place of stimulation could be due to the absence of ramping (leading to a broader spectrum of the acoustic signal at the onset), differences due to the higher carrier rate (900 pps instead of $100 \mathrm{pps}$ ), or the broader spectrum of the transposed stimulus.

The used transposed stimulus in principle has spectral components at all even multiples of the modulation frequency. It is therefore spectrally broader than the 1 oct wide filtered click train used by Francart et al. [2009]. The spectrum of an example transposed stimulus with $\mathrm{f}_{\mathrm{c}}=2400 \mathrm{~Hz}$ and $\mathrm{f}_{\mathrm{m}}=100 \mathrm{~Hz}$ is shown in figure 9. It has a main component at $f_{c}$ and main harmonics at $f_{c} \pm f_{m}$ and $f_{c} \pm 2 f_{m}$. It also has harmonics at $f_{c}$ plus or minus the even multiples of $f_{m}$. However, the first of these is already $25 \mathrm{~dB}$ lower in magnitude than the main component. Therefore, given the subjects' hearing thresholds (figure 1) and the used acoustic levels (table III), components further than $4 f_{m}$ from the centre frequency were not perceived.

level of the auditory nerve. One can, however, distinguish between fine structure and envelope at the level of the (acoustic) input signal. 


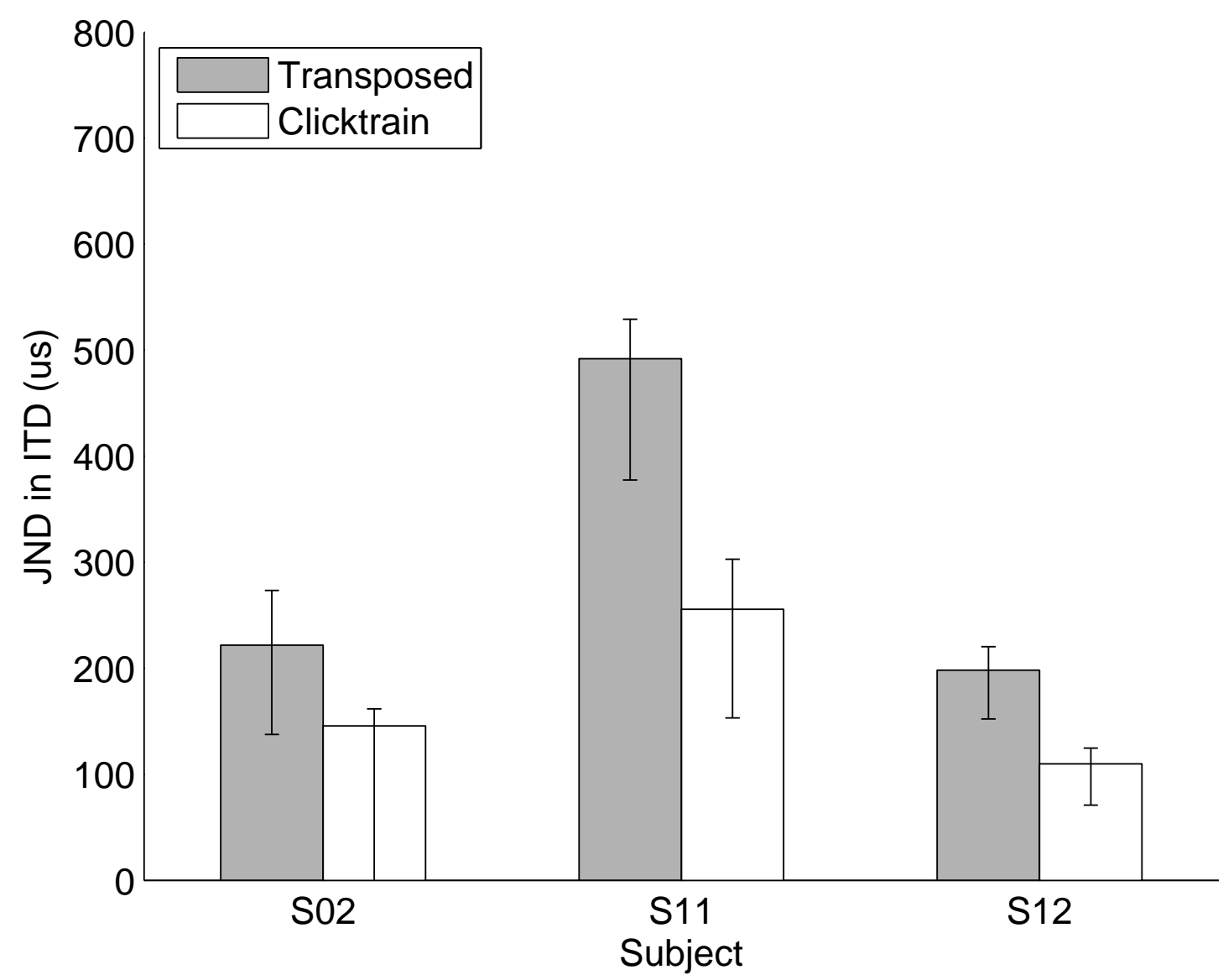

Figure 10: Comparison of ITD sensitivity with a transposed stimulus with $f_{m}=100, f_{c}=1200$ and with a 100 pps click train (results from Francart et al. [2009]).

Matching the place of excitation in the cochleas is not strictly required for sensitivity to ITD in NH subjects [Blanks et al., 2008, Nuetzel and Hafter, 1981]. In bilateral CI listeners the sensitivity to a mismatch in place of excitation is even smaller [Poon et al., 2009]. The sensitivity in the current study seems comparable to the sensitivity found in the latter study. This could be caused by broader auditory filters at the acoustic side due to severe hearing impairment, and spread of excitation at the electric side.

While no significant differences were found between carrier frequencies where the subjects were sensitive to ITD, there were inter-subject differences in the range of carrier frequencies for which the subjects were sensitive to ITD at all. For S02, this was between 1200 and 3600 Hz. For S11, this was between 600 and $3600 \mathrm{~Hz}$. For S12, this was between 300 and $2400 \mathrm{~Hz}$, and for S16 this was between 1200 and $2400 \mathrm{~Hz}$. While the upper limit can be explained by the amount of residual hearing at higher frequencies, the lower limit could be roughly indicative for the acoustic place of stimulation corresponding to the used electrode. In our previous study [Francart et al., 2009], we found corresponding acoustic frequency ranges of $1100-1600 \mathrm{~Hz}$ for S02,

$800-1600-3200 \mathrm{~Hz}$ for $\mathrm{S} 11$ and $400-800 \mathrm{~Hz}$ for $\mathrm{S} 12$. This is not incompatible with the results of the current experiment.

In figure 10 the current results with $\mathrm{f}_{\mathrm{m}}=100 \mathrm{~Hz}$ and $\mathrm{f}_{\mathrm{c}}=1200 \mathrm{~Hz}$ are compared to the click train results from Francart et al. [2009]. Note that the JND was recalculated for the latter results using the method described above, causing small deviations from the data reported by 


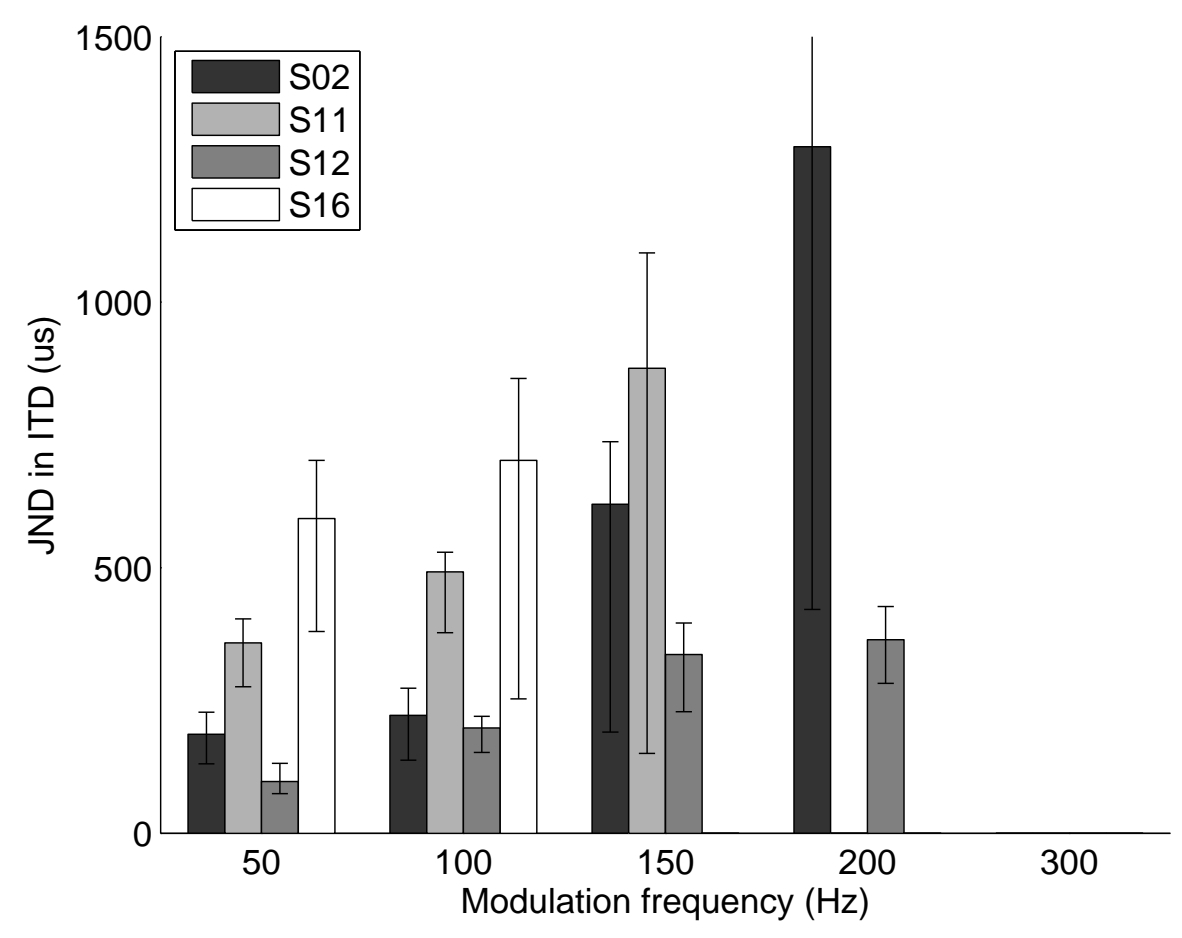

Figure 11: Results of experiment 2, the influence of modulation frequency on the JND in ITD for transposed stimuli $\left(\mathrm{f}_{\mathrm{c}}=1200 \mathrm{~Hz}\right)$. Missing bars indicate insensitivity to ITD.

Francart et al. [2009]. Performance with the current transposed stimulus was in the same order of magnitude as in our previous study. However, for S11 performance was significantly better for the click train. This corresponds to the results found with bilateral CIs in the literature [van Hoesel, 2007], where differences in sensitivity are mainly found for rates greater than $100 \mathrm{~Hz}$.

\subsection{Experiment 2}

In experiment 2 the influence of modulation frequency $\left(f_{m}\right)$ on ITD sensitivity was assessed. As the modulation frequency is only relevant when ongoing ITD cues are used (in contrast to onset/offset cues), we conducted some pilot experiments with S12, in which no onset/offset ramping was applied for stimuli with different modulation frequencies. This yielded good ITD sensitivity for modulation frequencies up to $400 \mathrm{~Hz}$. With $50 \mathrm{~ms}$ ramping, the results in figure 11 were obtained. This indicates that in our preliminary experiments the onset cue played an important role, and as it was largely eliminated by ramping, the results of the current experiment reflect mainly the use of ongoing ITD cues.

Figure 11 shows that ITD sensitivity was stable for 50 and $100 \mathrm{~Hz}$, but thereafter decreased with increasing $f_{m}$. There was a limit at around $150-200 \mathrm{~Hz}$ above which ITDs could not be detected.

From experiments with transposed stimuli with NH subjects, Bernstein and Trahiotis [2002] concluded that there is a limit of sensitivity to envelope ITD at around $250 \mathrm{~Hz}$. In their model, it is implemented as a second order low pass filter at $150 \mathrm{~Hz}$ and from comparison with data from the literature, they conclude that this is a limitation beyond the initial peripheral bandpass filtering. McFadden and Pasanen [1976], Nuetzel and Hafter [1981] found a similar effect for 


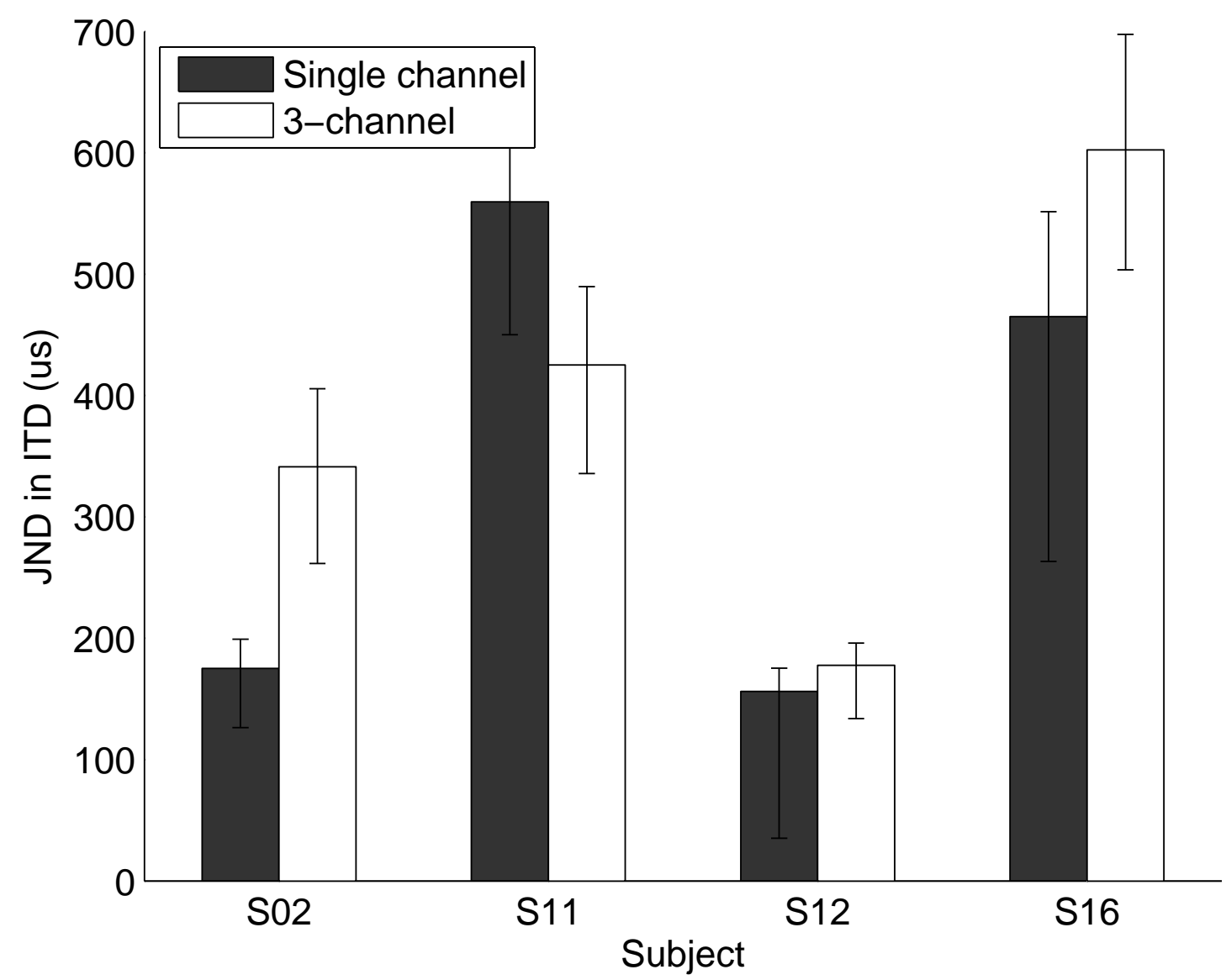

Figure 12: Results of experiment 3 compared to the results of experiment $1 . f_{c}=1200 \mathrm{~Hz}$ and $\mathrm{f}_{\mathrm{m}}=50 \mathrm{~Hz}$.

sinusoidally amplitude modulated stimuli. Similar observations were made by Griffin et al. [2005]

2 in neurophysiologic experiments with guinea pigs. These observations correspond well with our

3 data for bimodal listeners. This limitation may not be purely binaural. By means of

4 measurement of modulation detection thresholds for amplitude modulated sinusoids, several

5 studies [Ewert and Dau, 2000, Kohlrausch et al., 2000, Moore and Glasberg, 2001] have shown a

6 monaural rate limitation, with performance decreasing from $150 \mathrm{~Hz}$ onwards, functionally

7 independent of centre frequency.

8 In experiments with bilateral CIs, with unramped stimuli, a higher rate limit for ITD perception 9 was found by van Hoesel [2007] and van Hoesel et al. [2009]. In the latter study, when ramping

10 the onset of the stimulus, only 2 of the 6 listeners were still sensitive to ITD for a modulation

11 rate of $600 \mathrm{~Hz}$. In $\mathrm{NH}$ subjects, large inter-subject differences are also found for envelope ITD

12 sensitivity. Therefore in bilateral CI listeners probably the same envelope ITD rate limitation

13 may be present as found in NH listeners. Similarly to NH listeners, in CI listeners a monaural

14 rate limitation is also found for modulation detection [Shannon, 1992]. This is confirmed by

15 neurophysiologic recordings in the inferior colliculus in deaf cats implanted with a CI [Snyder

16 et al., 2000]. 
1

2

3

\section{$3.3 \quad$ Experiment 3}

In experiment 3 ITD sensitivity is measured for 3-electrode stimulation. The results are shown in figure 12. For comparison purposes the single-channel data at $50 \mathrm{~Hz}$ with a carrier frequency of $1200 \mathrm{~Hz}$ are also shown. Except for S02, there were no significant differences between single-channel and 3-channel stimulation.

When more than one channel is stimulated simultaneously, one could expect negative effects of channel interaction due to spread of excitation and/or - if the channels are perceptually distinguishable - effects similar to modulation detection interference, which is also found in CI listeners [Chatterjee and Oba, 2004, Richardson et al., 1998]. The results of the current study do not seem to reflect this finding.

Comparison of the results of the current experiment with those of bilateral CI listeners is difficult because we are not aware of any bilateral CI study that measures ITD sensitivity with multi-channel stimuli with direct stimulation. There are some studies that use clinical processors that stimulate multiple channels, but in that case it is impossible to separate the effects of the signal processing strategy and the stimulation of multiple channels per se.

There is a lot of evidence that in NH listeners, ITD information is combined across frequency channels (e.g., Trahiotis and Stern [1989], Trahiotis et al. [2005]). E.g., for high-frequency noise bands, greater extents of laterality are achieved for larger bandwidths [Trahiotis and Bernstein, 1986], and if contradictory information is present between different channels, ITD perception performance decreases [Dye, 1990]. If ambiguous information is present across channels, the auditory system combines information from the different channels [Trahiotis et al., 2005]. Therefore, on the one hand, if electric stimulation of a single electrode would provide ambiguous information (e.g., due to abnormally synchronous firing of nerve fibers), one could expect an improvement in performance with the addition of extra electrodes. If, on the other hand, contradictory information would be introduced, one would expect a decrease in performance. However, unless a negative and a positive effect cancelled each other out, neither effect seemed to be present in our data.

\section{General discussion and conclusions}

We have shown that bimodal listeners can be sensitive to ITDs in transposed stimuli. Performance for a certain electrode did not strongly depend on the carrier frequency of the acoustic signal. Performance decreased with increasing modulation frequency, with a limit of sensitivity at around $150-200 \mathrm{~Hz}$. Bimodal listeners were also sensitive to ITDs in a 3 -channel stimulus that was generated with signal processing similar to the clinical CIS processing. There was no effect of interference when stimulating multiple electrodes simultaneously, nor was there an additive effect.

While there is a rate limit for ITD perception, many vowels spoken by male speakers have a fundamental frequency lower than the found limit of around $150-200 \mathrm{~Hz}$, and for higher rates, if the onset is fast enough, ITDs can still be perceived. ITD perception could lead to improved sound source localization and binaural unmasking.

While the stimuli used in the current study are different from stimuli that would be generated by a clinical speech processor, they are very similar in the sense that (1) similar carrier rates are used, (2) a transposed stimulus is close to a half-wave rectified, low-pass filtered vowel, and (3) the 3 electrodes were tonotopically as close as possible together, and therefore represent a 
worst case scenario for channel interactions. The data of this study bridge the vast amount of single-electrode ITD data and the real ITD as could be perceived by CI patients using their clinical speech processor and contralateral HA. However, for optimal ITD perception with clinical systems, the speech processor and HA should be modified to be properly balanced in loudness, have similar loudness growth, are more or less matched in place of excitation, and are synchronized in time. Moreover, the speech processor should properly transmit envelope modulations that are synchronous between the channels (which is not necessarily the case with the current ACE processing scheme, mainly because of the maxima selection, the full-wave rectification and the filter bank design) and that have an envelope shape that enables optimal ITD perception. Our data indicate that under these circumstances ITD perception in a real-life environment should be possible with bimodal stimulation

\section{${ }_{12}$ Acknowledgements}

This research was supported by IWT-Vlaanderen and Cochlear, project 080304 . Additionally, Tom Francart was sponsored by a Post Doctoral Mandate (BOF) from the K.U.Leuven and Anneke Lenssen was sponsored by the EU-ITN project AUDIS. We are indebted to our test subjects who spent many hours of their valuable time enthusiastically participating in the experiments. We thank the staff of the audiology department of the hospital of Maastricht and the hospital of Leuven for their kind cooperation and flexibility.

\section{References}

L.R. Bernstein and C. Trahiotis. Enhancing sensitivity to interaural delays at high frequencies by using "transposed stimuli". J Acoust Soc Am, 112(3 Pt 1):1026-36, 2002.

L.R. Bernstein and C. Trahiotis. Lateralization of low-frequency, complex waveforms: the use of envelope-based temporal disparities. J Acoust Soc Am, 77(5):1868-80, 1985.

L.R. Bernstein and C. Trahiotis. How sensitivity to ongoing interaural temporal disparities is affected by manipulations of temporal features of the envelopes of high-frequency stimuli. $J$ Acoust Soc Am, 125(5):3234-42, 2009.

D.A. Blanks, J.M. Roberts, E. Buss, J. Hall, and D.C. Fitzpatrick. Neural and behavioral sensitivity to interaural time differences using amplitude modulated tones with mismatched carrier frequencies. J Assoc Res Otolaryngol, 8(3):393-408, 2007.

D.A. Blanks, E. Buss, J.H. Grose, D.C. Fitzpatrick, and J. Hall. Interaural time discrimination of envelopes carried on high-frequency tones as a function of level and interaural carrier mismatch. Ear Hear, 29(5):674-83, 2008.

T.N. Buell, S.J. Griffin, and L.R. Bernstein. Listeners' sensitivity to "onset/offset" and "ongoing" interaural delays in high-frequency, sinusoidally amplitude-modulated tones. J Acoust Soc Am, 123:279, 2008.

M. Chatterjee and S.I. Oba. Across- and within-channel envelope interactions in cochlear implant listeners. J Assoc Res Otolaryngol, 5(4):360-75, 2004.

T.Y. Ching, C. Psarros, M. Hill, H. Dillon, and P. Incerti. Should children who use cochlear implants wear hearing aids in the opposite ear? Ear Hear, 22(5):365-80, 2001.

T.Y. Ching, P. Incerti, and M. Hill. Binaural benefits for adults who use hearing aids and cochlear implants in opposite ears. Ear Hear, 25(1):9-21, 2004. 
T.Y. Ching, M. Hill, J. Brew, P. Incerti, S. Priolo, E. Rushbrook, and L. Forsythe. The effect of auditory experience on speech perception, localization, and functional performance of children who use a cochlear implant and a hearing aid in opposite ears. Int J Audiol, 44(12):677-90, 2005.

T.Y. Ching, E. van Wanrooy, and H. Dillon. Binaural-bimodal fitting or bilateral implantation for managing severe to profound deafness: a review. Trends Amplif, 11(3):161-92, 2007.

S. Colburn, B.G. Shinn-Cunningham, G.J. Kidd, and N. Durlach. The perceptual consequences of binaural hearing. Int $J$ Audiol, 45 Suppl 1:34-44, 2006.

8 R.H.J. Dye. The combination of interaural information across frequencies: lateralization on the basis of interaural delay. J Acoust Soc Am, 88(5):2159-70, 1990.

S.D. Ewert and T. Dau. Characterizing frequency selectivity for envelope fluctuations. J Acoust Soc Am, 108(3 Pt 1):1181-96, 2000.

S.D. Ewert, M. Dietz, M. Klein-Hennig, and V. Hohmann. Advances in Auditory Physiology, Psychophysics and Models; The role of envelope wave form, adaptation, and attacks in binaural perception. Springer, New York, 2009.

W.E. Feddersen, T.T. Sandel, D.C. Teas, and L. A. Jeffress. Localization of high-frequency tones. J Acoust Soc Am, 29(9):988-991, 1957.

T. Francart, J. Brokx, and J. Wouters. Sensitivity to interaural level difference and loudness growth with bilateral bimodal stimulation. Audiol Neurootol., 13(5):309-319, 2008a.

T. Francart, A. van Wieringen, and J. Wouters. APEX 3: a multi-purpose test platform for auditory psychophysical experiments. J Neurosci Methods, 172(2):283-293, 2008b.

T. Francart, J. Brokx, and J. Wouters. Sensitivity to interaural time differences with combined cochlear implant and acoustic stimulation. J Assoc Res Otolaryngol, 10(1):131-41, 2009.

D.W. Grantham, D.H. Ashmead, T.A. Ricketts, D.S. Haynes, and R.F. Labadie. Interaural time and level difference thresholds for acoustically presented signals in post-lingually deafened adults fitted with bilateral cochlear implants using cis processing. Ear Hear, 29(1):33-44, 2008.

S.J. Griffin, L.R. Bernstein, N.J. Ingham, and D. McAlpine. Neural sensitivity to interaural envelope delays in the inferior colliculus of the guinea pig. $J$ Neurophysiol, 93(6):3463-78, 2005.

G.B. Henning. Detectability of interaural delay in high-frequency complex waveforms. $J$ Acoust Soc Am, 55(1):84-90, 1974.

A. Kohlrausch, R. Fassel, and T. Dau. The influence of carrier level and frequency on modulation and beat-detection thresholds for sinusoidal carriers. J Acoust Soc Am, 108(2):723-34, 2000.

B. Laback, S.M. Pok, W.D. Baumgartner, W.A. Deutsch, and K. Schmid. Sensitivity to interaural level and envelope time differences of two bilateral cochlear implant listeners using clinical sound processors. Ear Hear, 25(5):488-500, 2004.

B. Laback, I. Zimmerman, and P. Majdak. Perception of interaural time differences in electric and acoustic hearing. In Proceedings of the International Symposium on Auditory and Audiological Research, 2009.

P. Majdak, B. Laback, and W.D. Baumgartner. Effects of interaural time differences in fine structure and envelope on lateral discrimination in electric hearing. J Acoust Soc Am, 120(4): 2190-201, 2006.

D. McFadden and E.G. Pasanen. Lateralization of high frequencies based on interaural time differences. J Acoust Soc Am, 59(3):634-9, 1976. 
B.C. Moore and B.R. Glasberg. Temporal modulation transfer functions obtained using sinusoidal carriers with normally hearing and hearing-impaired listeners. J Acoust Soc Am, 110 (2):1067-73, 2001.

4 J.M. Nuetzel and E.R. Hafter. Discrimination of interaural delays in complex waveforms:

5 Spectral effects. J Acoust Soc Am, 69(4):1112-1118, 1981.

6 B.B. Poon, D.K. Eddington, V. Noel, and H.S. Colburn. Sensitivity to interaural time difference 7 with bilateral cochlear implants: Development over time and effect of interaural electrode 8 spacing. J Acoust Soc Am, 126(2):806-15, 2009.

9 L.M. Richardson, P.A. Busby, and G.M. Clark. Modulation detection interference in cochlear 10 implant subjects. J Acoust Soc Am, 104(1):442-52, 1998.

11 B.U. Seeber and H. Fastl. Localization cues with bilateral cochlear implants. J Acoust Soc Am, 123(2):1030-42, 2008.

B.U. Seeber, U. Baumann, and H. Fastl. Localization ability with bimodal hearing aids and bilateral cochlear implants. J Acoust Soc Am, 116(3):1698-709, 2004.

P. Senn, M. Kompis, M. Vischer, and R. Haeusler. Minimum audible angle, just noticeable interaural differences and speech intelligibility with bilateral cochlear implants using clinical speech processors. Audiol Neurootol, 10(6):342-52, 2005.

R.V. Shannon. Temporal modulation transfer functions in patients with cochlear implants. $J$ Acoust Soc Am, 91(4 Pt 1):2156-64, 1992.

R.L. Snyder, M. Vollmer, C.M. Moore, S.J. Rebscher, P.A. Leake, and R.E. Beitel. Responses of inferior colliculus neurons to amplitude-modulated intracochlear electrical pulses in deaf cats. $J$ Neurophysiol, 84(1):166-83, 2000.

C. Trahiotis and L.R. Bernstein. Lateralization of bands of noise and sinusoidally amplitude-modulated tones: effects of spectral locus and bandwidth. J Acoust Soc Am, 79(6): 1950-7, 1986.

C. Trahiotis and R.M. Stern. Lateralization of bands of noise: effects of bandwidth and differences of interaural time and phase. J Acoust Soc Am, 86(4):1285-93, 1989.

C. Trahiotis, L.R. Bernstein, R.M. Stern, and T.N. Buell. Sound Source Localization; Interaural correlation as the basis of a working model of binaural processing: an introduction, chapter 7, pages 238-271. Springer, New York, 2005.

R.S. Tyler, C.C. Dunn, S.A. Witt, and W.G. Noble. Speech perception and localization with adults with bilateral sequential cochlear implants. Ear Hear, 28(2 Suppl):86S-90S, 2007.

S. van de Par and A. Kohlrausch. A new approach to comparing binaural masking level differences at low and high frequencies. J Acoust Soc Am, 101(3):1671-80, 1997.

R.J. van Hoesel. Sensitivity to binaural timing in bilateral cochlear implant users. J Acoust Soc Am, 121(4):2192-206, 2007.

R.J. van Hoesel. Exploring the benefits of bilateral cochlear implants. Audiol Neurootol, 9(4): 234-46, 2004.

R.J. van Hoesel and R.S. Tyler. Speech perception, localization, and lateralization with bilateral cochlear implants. J Acoust Soc Am, 113(3):1617-30, 2003.

R.J. van Hoesel, G.L. Jones, and R.Y. Litovsky. Interaural time-delay sensitivity in bilateral cochlear implant users: Effects of pulse rate, modulation rate, and place of stimulation. $J$ Assoc Res Otolaryngol, 2009. 
1 F.A. Wichmann and N.J. Hill. The psychometric function: II. Bootstrap-based confidence 2 intervals and sampling. Perception and Psychophysics, 63(8):1314-1329, 2001.

3 W.A. Yost. Discriminations of interaural phase differences. J Acoust Soc Am, 55(6):1299-303, 41974.

${ }_{5}$ W.A. Yost, F.L. Wightman, and David M. Green. Lateralization of filtered clicks. J Acoust Soc $6 \mathrm{Am}, 50(6 \mathrm{~B}): 1526-1531,1971$. 


\section{List of Tables}

I Subject information: "Age" is in years at the time of testing. "M of use" is the number of months of implant use at the time of testing. "CI side" is left (L) or right (R), the HA was on the other side. The two rightmost columns show the tested electrodes in respectively experiment 1 and 2 , and experiment $3 . \ldots \ldots$

II Stimulus parameter overview. $f_{m}$ is the modulation rate, "El carrier" is the carrier rate of the electric signal, $f_{c}$ is the carrier frequency of the acoustic signal. For experiment 3 , the given acoustic carrier frequencies, are the center frequencies of the analysis filter bank. A $\checkmark$ in "ramping" indicates that ramping of $50 \mathrm{~ms}$ was

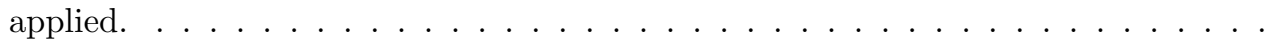

III Average broadband acoustic level per subject and stimulus type, all in dB SPL. . . 


\section{List of Figures}

1 Pure tone unaided audiograms as measured during routine audiometry. Note that the vertical axis starts at $50 \mathrm{dBHL} \ldots \ldots \ldots \ldots \ldots$

2 Fragment of an example transposed stimulus. The top panel shows the acoustic signal and the bottom panel shows the pulses on a single electrode. The modulation frequency $\mathrm{f}_{\mathrm{m}}=50 \mathrm{~Hz}$, the acoustic carrier frequency $\mathrm{f}_{\mathrm{c}}=300 \mathrm{~Hz}$ and the electric carrier was a pulse train of $900 \mathrm{pps} \ldots \ldots \ldots \ldots$

3 CIS-like signal processing, as used in experiment $3 \ldots \ldots \ldots \ldots$

4 Spectrum of the filtered click train and the frequency response of the three used band pass filters. . . . . . . . . . . . . . . . . . . . 10

$5 \quad$ Fragment of an example 3-channel stimulus, as used in experiment 3. The top panel shows the acoustic signal. The bottom panel shows the electric signal. Only the magnitude of the pulses is shown. . . . . . . . . . . . . . . . . .

6 Example psychometric function. In this case the interaural delay for a centered percept was $1208 \mu$ s and the JND was $362 \mu \mathrm{s} . ~ \ldots \ldots \ldots \ldots$. . . . . . . . .

$7 \quad$ Median delay of the electric signal needed to compensate for the acoustic travelling wave delay $(D)$. The results were pooled per subject over experiment $1 / 2$ and 3 .

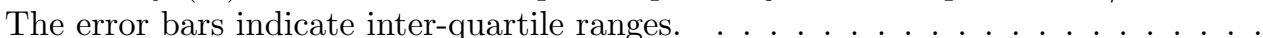

8 Results of experiment 1 . The modulation frequency $\mathrm{f}_{\mathrm{m}}=50 \mathrm{~Hz}$ and the electric carrier was a pulse train of 900 pps. The carrier frequency $\left(f_{c}\right)$ is shown in the inset. 16

$9 \quad$ Spectrum of an example transposed stimulus with $\mathrm{f}_{\mathrm{c}}=2400 \mathrm{~Hz}$ and $\mathrm{f}_{\mathrm{m}}=100 \mathrm{~Hz} . \quad 17$

10 Comparison of ITD sensitivity with a transposed stimulus with $f_{m}=100, f_{c}=1200$ and with a 100 pps click train (results from Francart et al. [2009]). . . . . . . . 18

11 Results of experiment 2, the influence of modulation frequency on the JND in ITD for transposed stimuli $\left(\mathrm{f}_{\mathrm{c}}=1200 \mathrm{~Hz}\right)$. Missing bars indicate insensitivity to ITD. .

12 Results of experiment 3 compared to the results of experiment $1 . f_{c}=1200 \mathrm{~Hz}$ and

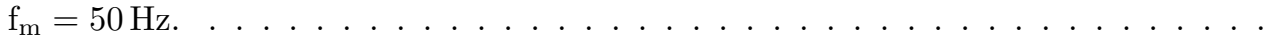

